\title{
1.1. Quench Analysis of the DEMO CS1 Coil
}

\section{Aleksandra Dembkowska ${ }^{1}$, Monika Lewandowska ${ }^{1 *}$, Xabier Sarasola $^{2}$, Kamil Sedlak $^{2}$}

* Corresponding author, monika.lewandowska@zut.edu.pl

${ }^{1}$ Faculty of Mechanical Engineering and Mechatronics, West Pomeranian University of Technology, Szczecin, Al. Piastów 19, 70-311 Szczecin, Poland

2 École Polytechnique Fédérale de Lausanne (EPFL), Swiss Plasma Center (SPC), CH-5232 Villigen PSI, Switzerland

\begin{abstract}
The European DEMOnstration Fusion Power Plant (EU-DEMO) is being designed as an intermediary stage between the ITER experimental reactor and future commercial fusion power plants. The EUDEMO is based on the tokamak concept with a fully superconducting magnet system. The Central Solenoid (CS) of the EU-DEMO will consist of five modules, namely: CSU3, CSU2, CS1, CSL2 and CSL3, located vertically one above the other. The central CS1 module will be subjected to the most demanding operating conditions (the highest magnetic field and mechanical loads). Two concepts of the CS1 winding pack (WP) are being designed by CEA IRFM (France) and EPFL-SPC (Switzerland) teams. The pancake wound WP proposed by CEA is based on Wind \& React $\mathrm{Nb}_{3} \mathrm{Sn}$ Cable-in-Conduit Conductor, whereas the hybrid WP developed by EPFL-SPC consist of 10 sub-coils, layer-wound using: HTS (RE-123), React \& Wind $\mathrm{Nb}_{3} \mathrm{Sn}$ and NbTi conductors in the high, medium and low field sections, respectively. Each design iteration undergoes comprehensive electromagnetic, mechanical and thermalhydraulic analyses aimed at verification if it fulfils the design performance criteria. Our present work is focused on the quench analysis of all the hybrid CS1 sub-coils, aimed at the assessment of the maximum hot-spot temperature. The analysis, based on the iteration of the hybrid design proposed in 2017, is performed using the THEA CryoSoft code. We assume that quench is initiated at the beginning of premagnetization phase and include in our model the realistic magnetic field distribution along each conductor computed with 2D axi-symmetrical finite element model in ANSYS. We study the effect of taking into account heat transfer between neighbouring turns and heat generation due to AC losses during the fast discharge on the value of the hot spot temperature.
\end{abstract}

Keywords: quench; hot spot temperature; CS coil; EU-DEMO; thermal-hydraulic phenomena; design and analysis

\section{Introduction}

Comprehensive conceptual studies on the European DEMOnstration Fusion Power Plant (EUDEMO), designed with the aim of generating several hundred MWs of net electricity from fusion power, are carried out by the EUROfusion consortium [1-3]. Extensive design and assessment works on the EU-DEMO magnet system were mainly focused on the Toroidal Field (TF) coil, recently first proposals for the Central Solenoid (CS) and Poloidal Field coils were developed, as reviewed in [4-6]. The CS coil of the EU-DEMO tokamak will consist of five modules located vertically one above the other, namely CSU3, CSU2, CS1, CSL2 and CSL3, the central of which (CS1) will be subjected to the highest magnetic field and mechanical loads. The double-pancake wound winding pack (WP) design of the CS1 module based on Wind \& React $\mathrm{Nb}_{3} \mathrm{Sn}$ conductor has been proposed by CEA IRFM [7-10], whereas a hybrid CS1 WP design is being developed by the EPFL-SPC team $[11,12]$. 
Previous studies [11] indicated that the use of High $T_{c}$ Superconductors (HTS) in the highest field allows achieving the required magnetic flux in the EU-DEMO CS at a reduced outer diameter, which would enable a reduced overall size and cost of EU-DEMO. The WP design of the CS1 module proposed by EPFL-SPC, using a superconductor grading, is based on 10 sub-coils (SC). Each of SC is composed of two layers wound using the same conductor. HTS (Re-123), React \& Wind $\mathrm{Nb}_{3} \mathrm{Sn}$ and $\mathrm{NbTi}$ conductors are used in the high (SCs 1-2), medium (SCs 3-7) and low field (SCs 8-10) sections, respectively. Thus, the CS1 module consists of 20 layers (Ls). In contrast to an earlier iterations of the design [11], the design considered in the present study [12], based on the 2015 DEMO reference, took into account the vertical mechanical loads and graded the stainless steel fraction across the winding pack, including a co-wound strip of steel in the two innermost sub-coils (see Fig. 1a and b). The thermalhydraulic study of the normal operation of CS1 conductors during the full plasma cycle, aimed at the assessment of the minimum temperature margin, was reported in [13-16]. In the present paper we discuss the preliminary quench analysis of the recent iteration of the CS1 design [12], focused on estimation of the maximum quench hot spot temperature, verification if it fulfills the performance criterion of $150 \mathrm{~K}$, and identification of the effect of different thermal-hydraulic phenomena on the value of the hot-spot temperature.
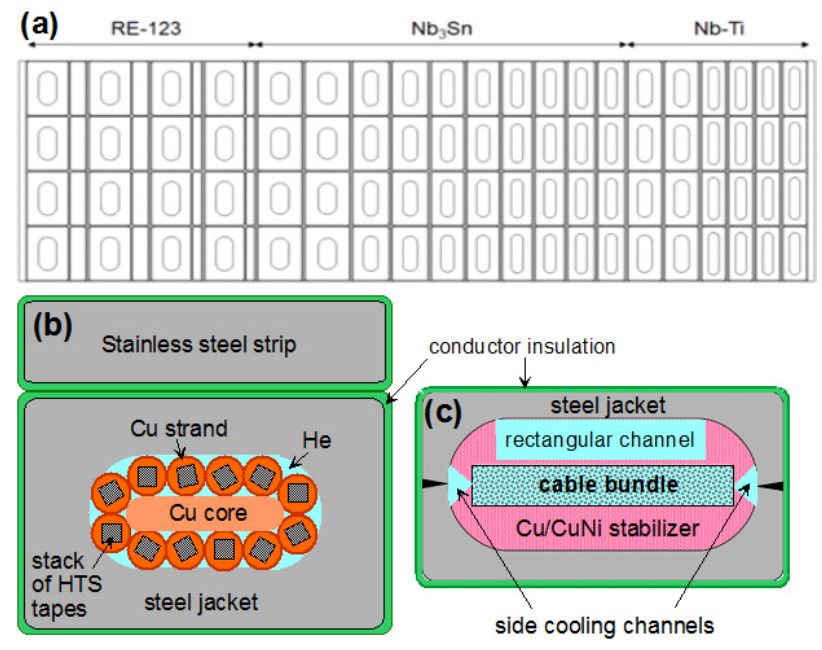

Figure 1. (a) Layout of the four conductor rows in the CS1 WP and schematic cross section of a (b) HTS and (c) LTS conductor.

\section{Basic assumptions}

The conductors designed for each SC are optimized, i.e. the amount of superconductor, copper, steel and helium is different in different SC, depending on the magnetic field, as well as heat and mechanical electromagnetic loads expected in a given SC during normal operation. Schematic layout of a HTS and a Low $T_{c}$ Superconductor (LTS) CS1 conductors is shown in Fig. $1 \mathrm{~b}$ and c. The geometrical characteristics of all CS1 conductors relevant for the present analysis is discussed in detail in [16]. We use the scaling relations and parameters for the critical current densities of superconducting materials taken from [11], which allow to calculate the current sharing temperature $T_{c s}(B, I)$ in each conductor. From the hydraulic point of view all CS1 conductors are connected in parallel and they are cooled by forced flow of supercritical helium (SHe). The length of conductors varies in the range from $944 \mathrm{~m}(\mathrm{~L} 1)$ to $1371 \mathrm{~m}$ (L20). We assume that the SHe inlet pressure and temperature are: $p_{i n}=0.6 \mathrm{MPa}$ and $T_{i n}=4.5 \mathrm{~K}$, whereas the expected pressure drop in conductors at normal operation is $0.1 \mathrm{MPa}$.

The plasma scenario considered in $[7,13,14]$, also retained as a reference for the present analysis, includes the following main phases: $10 \mathrm{~s}$ premagnetisation (Premag), $80 \mathrm{~s}$ plasma current ramp-up, 
$7200 \mathrm{~s}$ plasma burn (between the Start of Flat Top (SOF) and the End of Flat Top (EOF)) and $600 \mathrm{~s}$ dwell. In the Premag phase both magnetic field profile and the operating current in CS1 conductors remain constant and they reach their maximum values $\left(I_{\text {Premag }}=50.95 \mathrm{kA}\right.$, while the proportion $I_{\text {Premag }}$ : $I_{S O F}: I_{E O F}=50.95 \mathrm{kA}:-7.84 \mathrm{kA}:-50.95 \mathrm{kA}$, which is equal to the respective proportion of the CS1 module total currents 57.14 MAt: -8.79 MAt :-57.14 MAt in the DEMO 2015 reference design [12]). Thus, the Premag also features low values of the temperature margin profile in each conductor, defined as:

$$
\Delta T_{\text {marg }}(x, t)=T_{c s}(x, t)-T_{\text {scon }}(x, t),
$$

where $x$ is the coordinate along a conductor, and $T_{\text {scon }}$ is the superconductor operating temperature. Such conditions are favorable for quench occurrence.

In our previous studies $[13,14,16]$, we observed that the global minimum of $\Delta T_{\text {marg }}$ for all CS1 conductors was typically achieved either during the fast breakdown just after the end of the Premag phase, or in the last second of the dwell phase, just before the start of next Premag. In all conductors the global minimum of $\Delta T_{\text {marg }}$ was only slightly smaller than the respective minimum $\Delta T_{\text {marg }}$ observed in the Premag phase. For simplification, our present analysis is focused on the case where quench is initiated at the beginning of the Premag phase.

We performed quench simulations using the THEA code by CryoSoft [17] for the outer conductor of each SC (even layers). Behavior of each conductor was studied separately, i.e. inter-layer heat transfer was not taken into account. The THEA model of each conductor includes several parallel 1D thermal (solid) and hydraulic (fluid) components, as presented schematically in Fig. 2.

In the THEA code thermal coupling between the thermal (solid) conductor components is expressed using the matrix of thermal resistances $R=1 /\left(h \cdot P_{\mathrm{c}}\right)$, where $P_{c}$ is the contact perimeter and $h$ is the heat transfer coefficient (HTC). In the HTS conductors, the strands - core and strands jacket contact perimeter is assumed equal to $n_{\text {strands }} \cdot 0.5 \mathrm{~mm}$. In the LTS conductors, the strands - stabilizer contact perimeter is assumed to be $15 \%$ of the cable space perimeter. Conservative assumptions about thermal-hydraulic

(a)
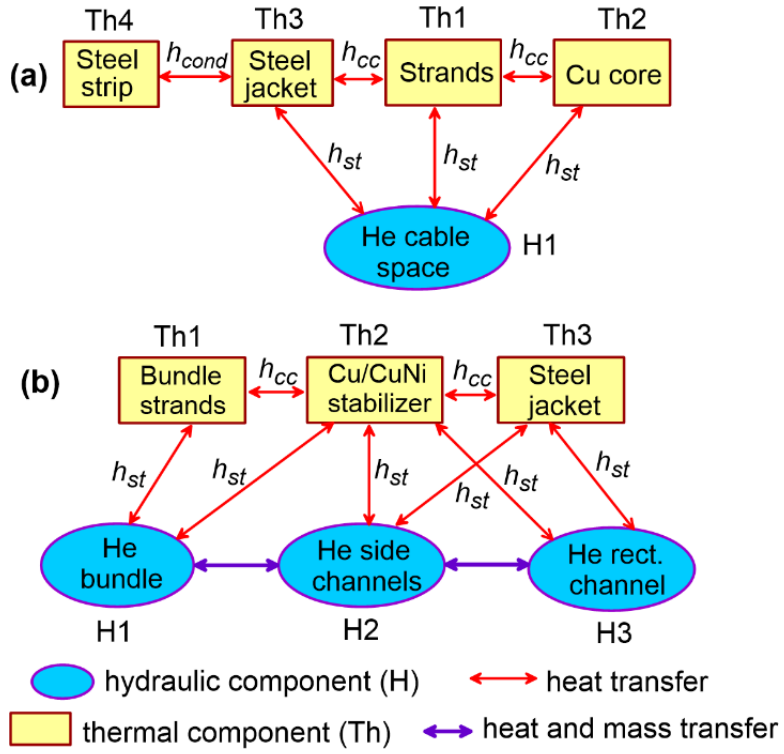

Figure 2. THEA model of (a) a HTS and (b) a LTS conductor, and thermal links between different conductor components. 
conductors' characteristics follow the recommendations agreed within the EUROfusion Magnet System (WPMAG) project team [18,19], which were also used e.g. in [7, 13-16]. The HTC between the fluid and solid components $\left(h_{s t}\right)$ is obtained with standard smooth tube correlations [20], the HTC due to the contact conductance between the solid components is conservatively assumed to be $h_{c c}=500 \mathrm{~W} /\left(\mathrm{m}^{2} \mathrm{~K}\right)$ [21]. Friction factor in cooling channels and in bundle regions of LTS cables is calculated with the Bhatti-Shah correlation [20] and the correlation developed in [22], respectively. Friction factor in HTS cables is obtained with the correlation suggested in [23]. We assumed that in the LTS conductors there are small gaps between the jacket and the $\mathrm{Cu} / \mathrm{CuNi}$ stabilizer and between the two profiles of the $\mathrm{Cu} / \mathrm{CuNi}$ stabilizer which allow helium exchange between the cooling channels and the bundle.

The adiabatic and fixed pressure (infinite reservoir) boundary conditions are imposed at both ends of each conductor. Two cases are considered concerning the inter-turn (IT) heat transfer: adiabatic conductor (no IT coupling) and with IT heat conduction between jackets of adjacent turns through the two layers of $1 \mathrm{~mm}$ thick conductor insulation, characterized by the HTC:

$$
h_{\text {cond }}=\lambda\left(T_{r e f}\right) / d \text {, }
$$

where $d=2 \mathrm{~mm}, \lambda$ is the thermal conductivity of the conductor insulation (assumed to be glassfiber/epoxy resin composite) and $T_{r e f}$ is the reference temperature equal to the average temperature of jackets in adjacent turns at a given location.

The maps of the radial and vertical component of the magnetic field along each conductor for the Premag phase were obtained using the 2D axi-symmetrical finite element model in ANSYS [24]. We took into account contributions generated by all the CS modules as well as by the Poloidal Field coils. The examples of resulting profiles of the total magnetic field are shown in Fig. 3.

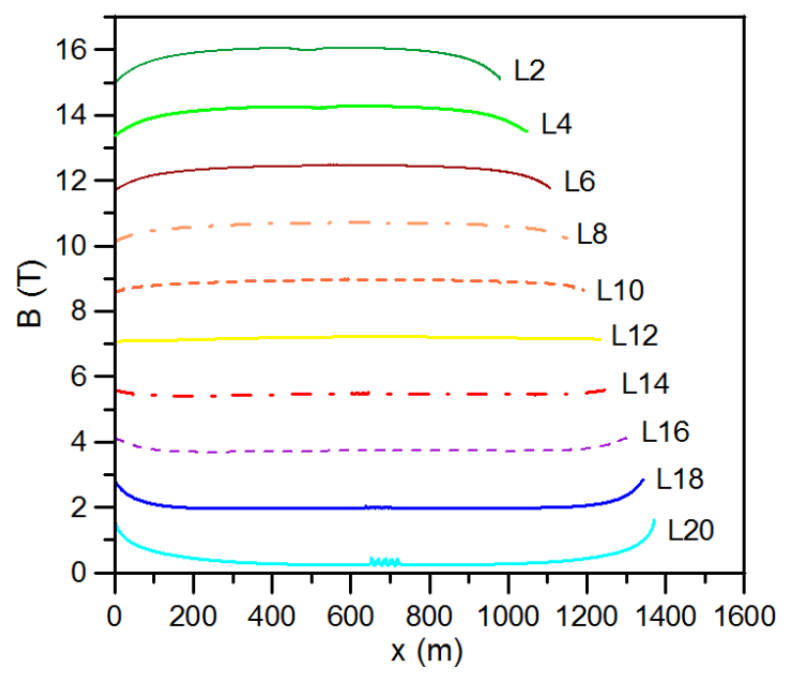

Figure 3. Computed magnetic field distribution in the outer conductor of each SC of CS1 (even layers) during the Premag phase.

At first simulations of normal operation during the Premag phase for each conductor were performed, assuming no heat load, with the current and the magnetic field profiles set equal to their values in the Premag, starting from the constant initial conditions: $T(x)=T_{i n}, p(x)=p_{\text {in }}$ until the steady state was reached. The steady state temperature, pressure and mass flow rate profiles along each conductor were saved. The obtained $\Delta T_{\text {marg }}$ profiles along the considered conductors in the Premag phase are shown in Fig. 4. It is seen that $\Delta T_{\text {marg }}$ values in all conductors are well above the $1.5 \mathrm{~K}$ performance criterion $[4,5]$, 
particularly in HTS layers (L2, L4). It is suggested in [18] to initiate quench either in a location where $\Delta T_{\text {marg }}$ is the smallest, i.e. where quench is most likely to occur, or in a region where $\Delta T_{\text {marg }}$ is the largest, since this case typically provides the highest value of the hot-spot temperature, thus can be considered the most conservative. However, in the CS1 conductors either the minimum (L14 - L20) or the maximum (L2 - L12) of $\Delta T_{\text {marg }}$ is located at the conductor boundary (see Fig. 4). Quench initiated at the conductor edge cannot be modelled very reliably, particularly taking into account simplified boundary conditions used in our study. In this situation, we decided to impose the heat pulse initiating quench at the location of $\Delta T_{\text {marg }}$ minimum in layers L2 - L12, or of $\Delta T_{\text {marg }}$ maximum in layers L14 - L20, so that in all cases the heat pulse was imposed far from the conductor boundary.

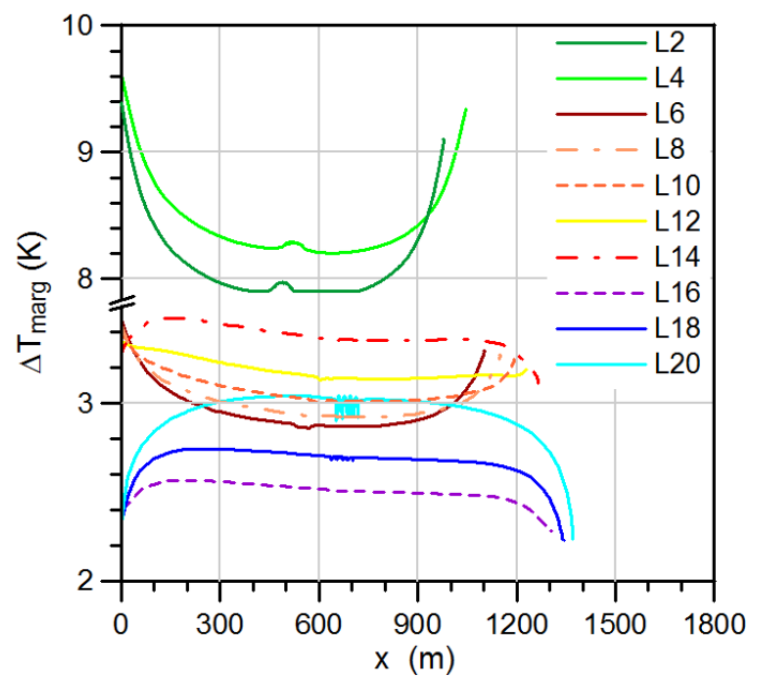

Figure 4. Temperature margin profiles in the CS1 conductors (even layers) during the Premag phase.

In the second stage of analysis quench simulations for each conductor were carried out, using the steady state temperature, pressure and mass flow rate profiles obtained earlier as the initial conditions. Quench was initiated at the beginning of the simulation $(t=0 \mathrm{~s})$ by a heat pulse of length $10 \mathrm{~cm}$ and duration $100 \mathrm{~ms}$, and energy 2 x MQE (Minimum Quench Energy) [18]. MQE was evaluated iteratively for the case with IT heat transfer. The quench detection voltage threshold was set at $0.1 \mathrm{~V}$. The current dump, assumed exponential with a characteristic time $\tau_{\text {dump }}=19.5 \mathrm{~s}$, starts with an additional delay $t_{\text {delay }}$ $=1.1 \mathrm{~s}$ due to the quench protection system reaction time:

$$
I(t)=\left\{\begin{array}{cc}
I_{\text {Premag }} & \text { for } t<t_{\text {dump }} \\
I_{\text {Premag }} \exp \left(-\frac{t-t_{\text {dump }}}{\tau_{\text {dump }}}\right) & \text { for } t \geq t_{\text {dump }}
\end{array},\right.
$$

where $t_{\text {dump }}=t_{\text {detection }}+t_{\text {delay }}$ is time in which the current dump starts. During the fast discharge the magnetic field decreases proportionally to the operating current, which induces heat generation (in $\mathrm{W} / \mathrm{m}$ ) due to AC coupling losses, assumed to follow the simple behaviour [25]:

$$
P_{\text {coupling }}(x, t)=\left\{\begin{array}{cl}
0 & \text { for } t<t_{\text {dump }} \\
\frac{n \tau S}{\mu_{0}}\left[\frac{B_{\text {Premag }}(x)}{\tau_{\text {dump }}} \exp \left(-\frac{t-t_{\text {dump }}}{\tau_{\text {dump }}}\right)\right]^{2} & \text { for } t \geq t_{\text {dump }}
\end{array},\right.
$$


where $n$ is the shape factor, $\tau$ is a time constant, and $S$ is the conductor cross section (excluding helium and steel jacket). The CS1 conductors designed by EPFL-SPC team have not yet been fully characterized in terms of AC losses. As agreed with the WPMAG project team, in the present analysis we used $n \tau=$ $75 \mathrm{~ms}$ specified for the ITER CS conductor [26], which is considered, for the time being, as a target value for the EU-DEMO conductors designers. It results from Eq. (4), that the power of the coupling losses it the highest at the very beginning of the fast discharge $\left(t=t_{\text {dump }}\right)$ and that the maximum value of $P_{\text {coupling }}$ in each conductor, equal to $n \tau S\left[\max \left(B_{\text {Premag }}(x)\right) / \tau_{\text {dump }}\right]^{2} / \mu_{0}$ (see some examples in Table 1 ), falls at the location of the maximum of the respective magnetic field profile (see Fig. 5a-b). It is seen that in each conductor $P_{\text {coupling }}$ values decrease about 1.7 / 7.8 times during the first $5 \mathrm{~s} / 20 \mathrm{~s}$ of current dump, respectively.
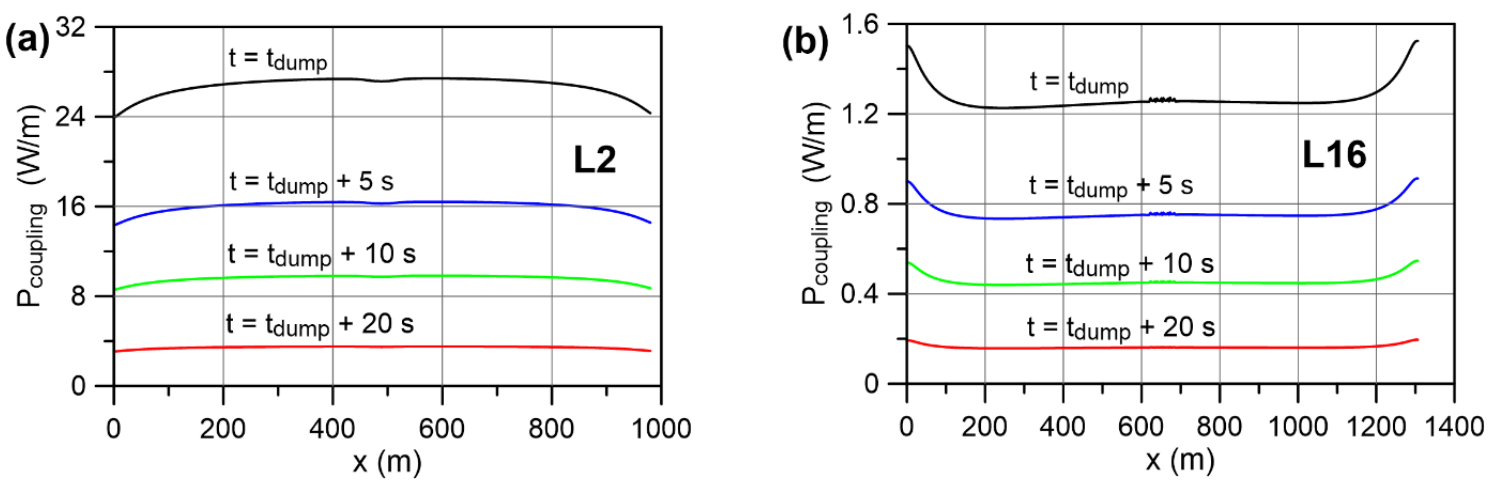

Figure 5. Examples of time evolution of the $P_{\text {coupling }}$ profile along the (a) L2 conductor, and (b) L16 conductor during the fast discharge.

In quench simulations we used a refined initial mesh, (with the refined region around the disturbance location) with automatic adaptivity (AdaptivityMethod Quench Thermal 1). Since quench simulations in full size HTS conductors have hardly ever been discussed in literature and they may be problematic [27], a special attention was given to the layer L2, for which the simulation was repeated several times using various values of parameters MinimumSize, MaximumSize, MinimumStep, and MaximumStep in order to make sure that the obtained results were not sensitive to the choice of the maximum and minimum time and space step.

\section{Results and discussion}

The maximum values of the bundle strands and jacket temperatures obtained in our quench simulations are shown in Fig. 6. In all considered CS1 layers the hot-spot acceptance temperature criterion specified in [26], i.e. $T_{\text {jacket } \max }<150 \mathrm{~K}$ is fulfilled with a large safety margin, however the maximum quench temperature of strands in all $\mathrm{Nb}_{3} \mathrm{Sn}$ layers (L6-L14) is above $150 \mathrm{~K}$, which calls for attention of the WP designers. Taking into account IT heat transfer does not affect significantly the obtained results - the hot-spot temperatures in the jacket for the case without IT is typically slightly $(0.6$ $-8 \mathrm{~K}$ ) higher than for the case with IT. Such a clear trend cannot be noticed in the hot-spot temperatures of bundle strands, which requires further clarification. 


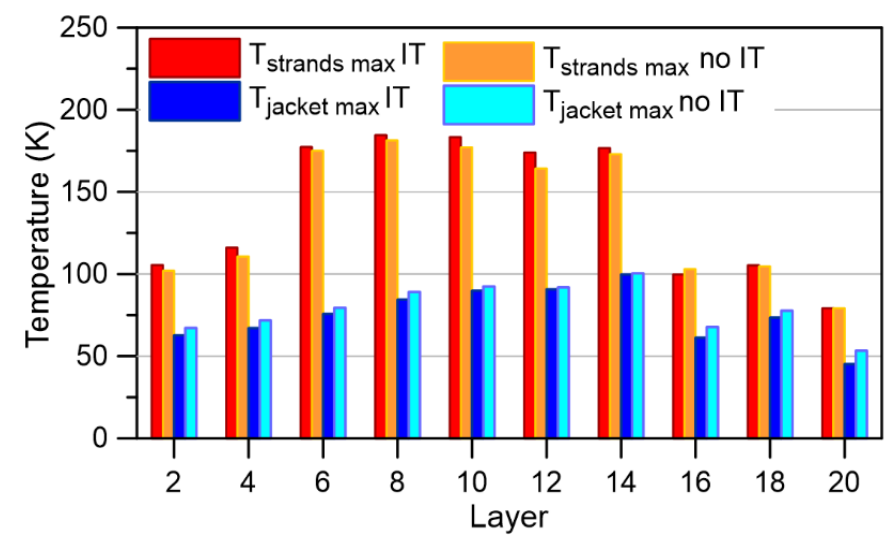

Figure 6. Maximum quench temperature in different thermal components of each considered layers (AC losses during the fast discharge were taken into account).

To understand the effect of IT heat transfer and heat generation due to AC coupling losses during the current dump on the evolution of the temperature field in conductors we selected three of the considered layers (L2, L6 and L16, made of $\mathrm{HTS}, \mathrm{Nb}_{3} \mathrm{Sn}$ and $\mathrm{NbTi}$, respectively), with the largest AC losses for a given superconducting material and we performed for them detailed comparison of the three following cases:

- Case A in which both AC losses and IT were taken into account,

- Case B with AC losses and without IT,

- Case C with no AC losses and no IT.

Table 1 and Figs. 7-10 show the summary of this comparison. It can be noticed that different layers exhibit quite different behavior, e.g. the maximum strands temperature is the highest in Case C in L2, in Case A in L6, and in Case B in L16. To clarify this issue we studied in detail the evolution of the temperature, pressure and mass flow rate profiles along the L2, L6 and L16 conductors (see Figs. 1119 ) and we identified several phenomena which may affect the value of the hot-spot temperature.

Table 1. Comparison of the main results obtained for layers L2, L6 and L16 in different in different cases discussed in text

\begin{tabular}{lrrrrrrrrr}
\hline & L2 & \multicolumn{1}{c}{ L2 } & L2 & L6 & L6 & L6 & L16 & L16 & L16 \\
& Case A & Case B & Case C & Case A & Case B & Case C & Case A & Case B & Case C \\
\hline$P_{\text {coupling }}^{\max }(\mathrm{W} / \mathrm{m})$ & 27.4 & 27.4 & - & 13.2 & 13.2 & - & 1.5 & 1.5 & - \\
$t_{\text {dump }}(\mathrm{s})$ & 6.21 & 6.28 & 6.28 & 2.67 & 2.68 & 2.68 & 6.20 & 6.28 & 6.28 \\
$T_{\text {jacket } \max }(\mathrm{K})$ & 62.7 & 67.2 & $\mathbf{7 0 . 8}$ & 75.6 & $\mathbf{7 9 . 5}$ & 77.0 & 61.2 & $\mathbf{6 7 . 8}$ & 66.1 \\
$T_{\text {strands max }}(\mathrm{K})$ & 105.5 & 102.1 & $\mathbf{1 1 3 . 4}$ & $\mathbf{1 7 7 . 4}$ & 175.1 & 167.6 & 99.7 & $\mathbf{1 0 3 . 0}$ & 99.1 \\
\hline
\end{tabular}



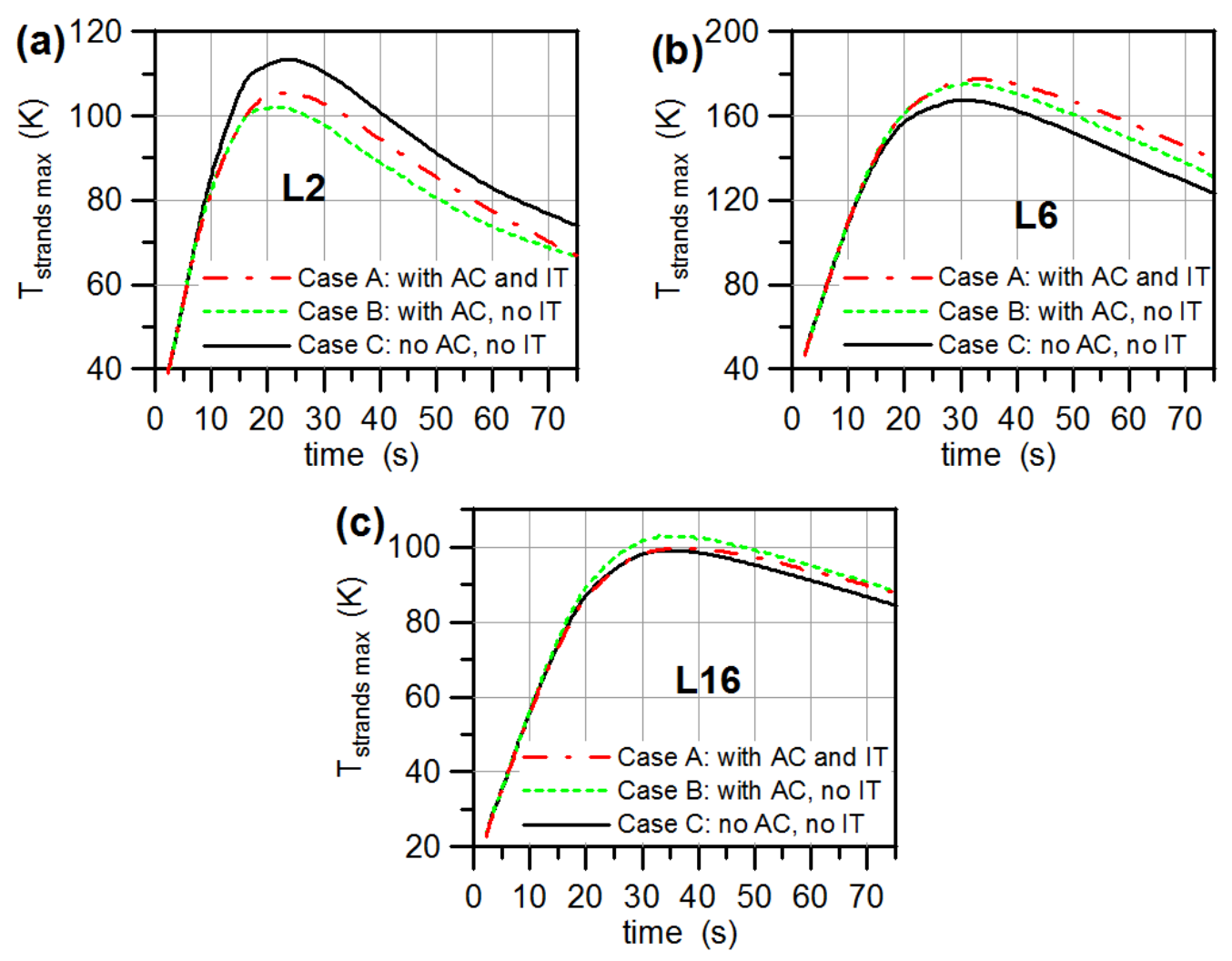

Figure 7. Time evolution of the maximum strands temperature during quench in different CS1 layers.
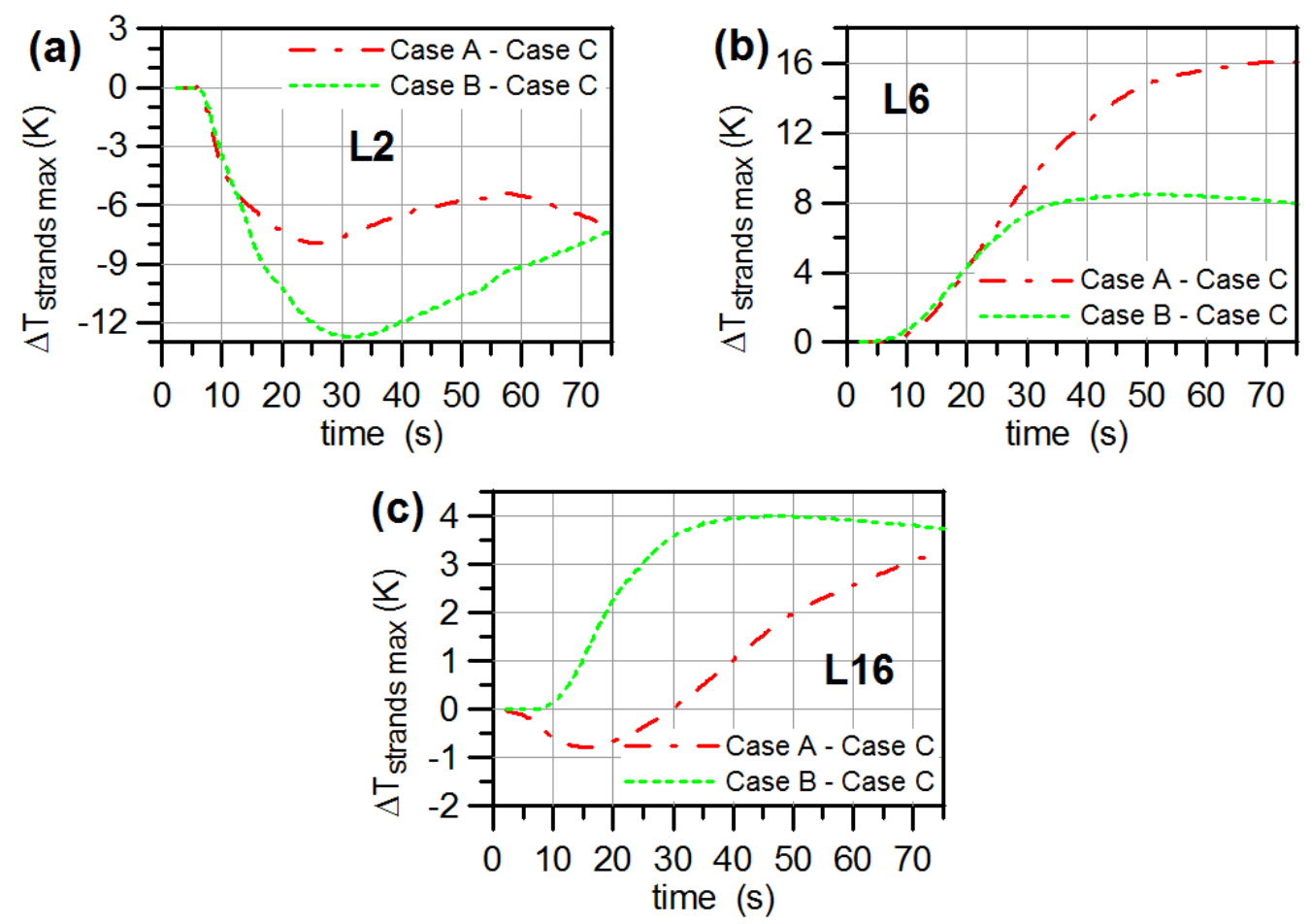

Figure 8. Time evolution of differences between the maximum strands temperatures during quench obtained in different cases discussed in text (with Case $\mathrm{C}$ serving as a reference). 

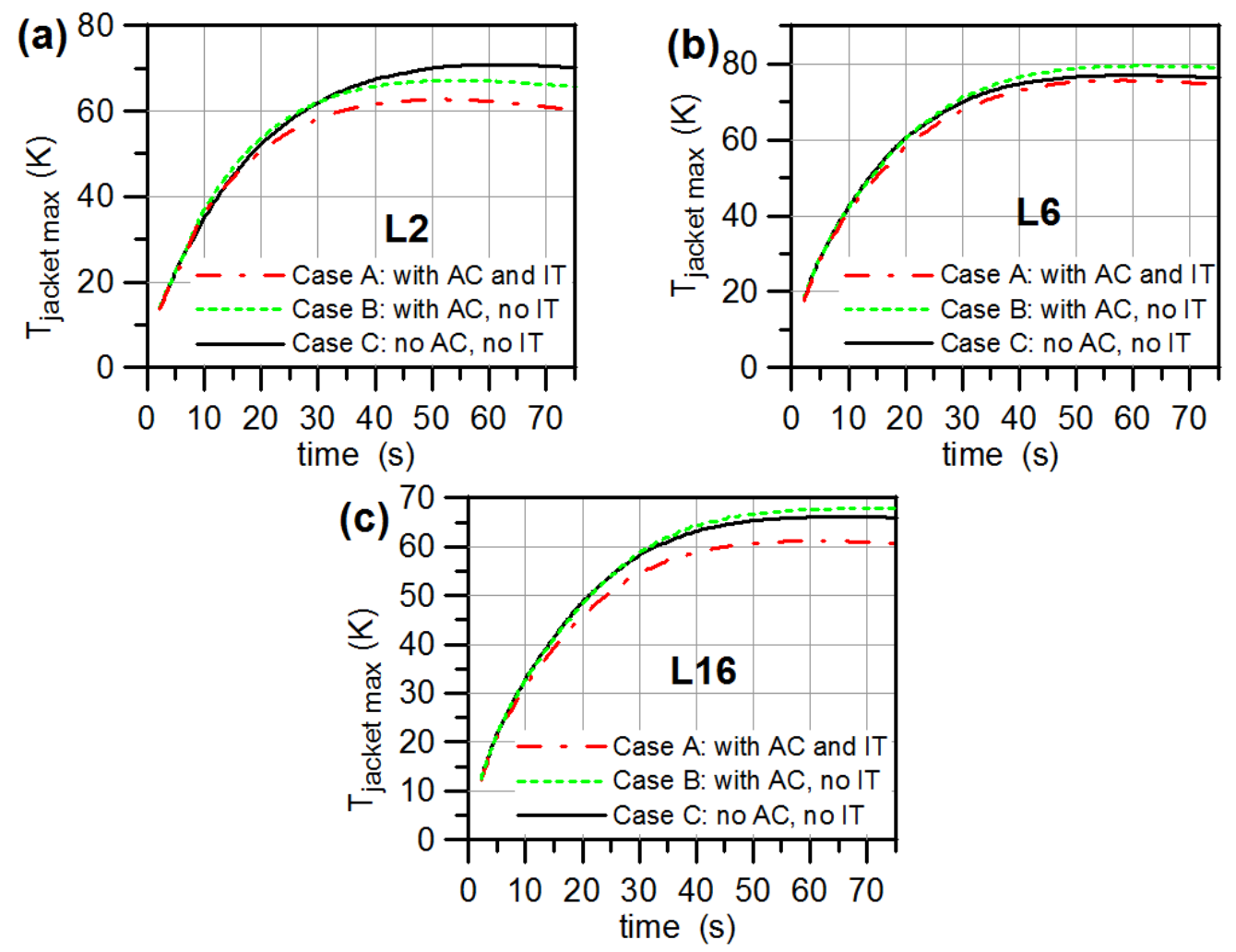

Figure 9. Time evolution of the maximum jacket temperature during quench in different CS1 layers.
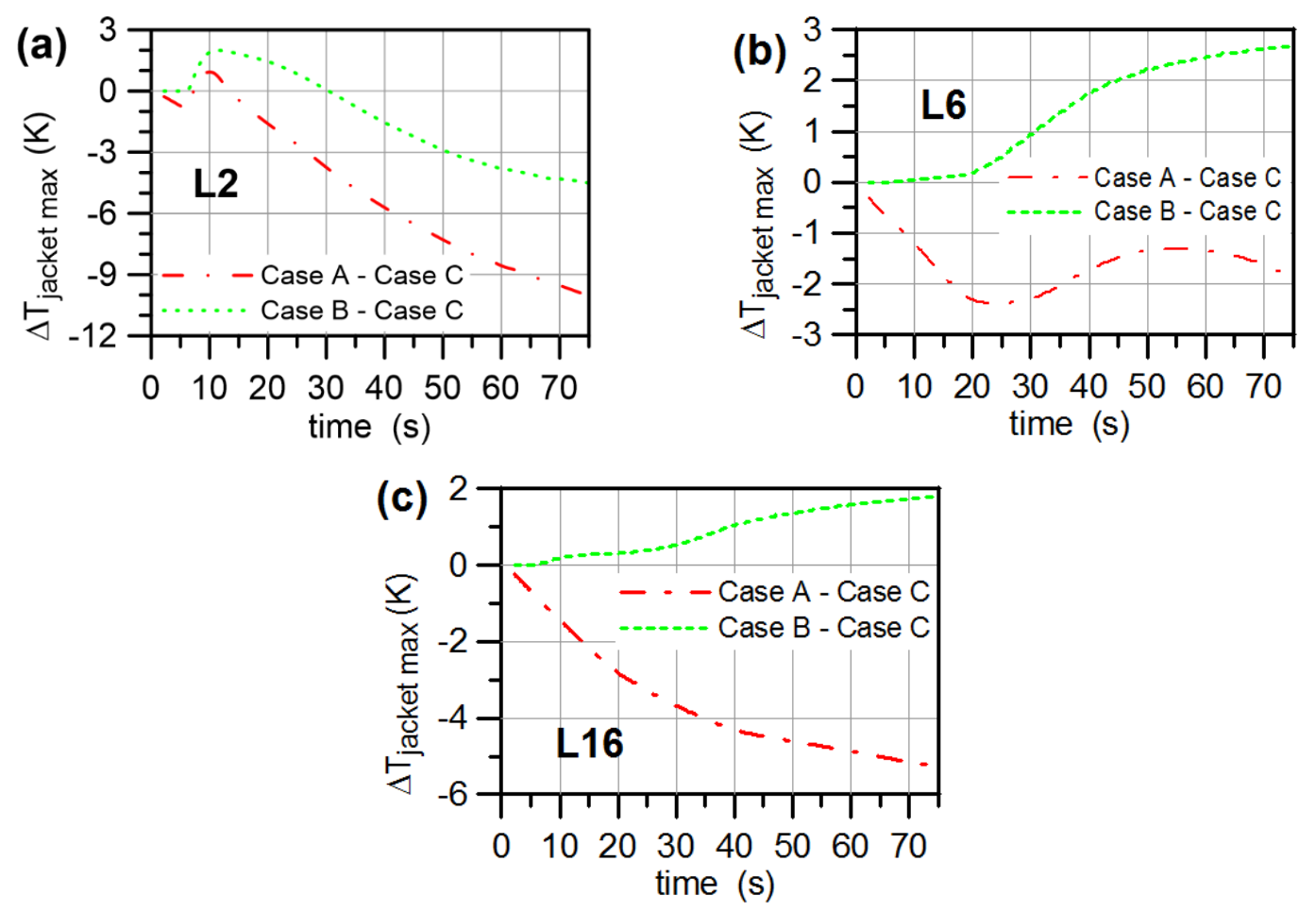

Figure 10. Time evolution of differences between the maximum jacket temperatures during quench obtained in different cases discussed in text (with Case $\mathrm{C}$ serving as a reference). 

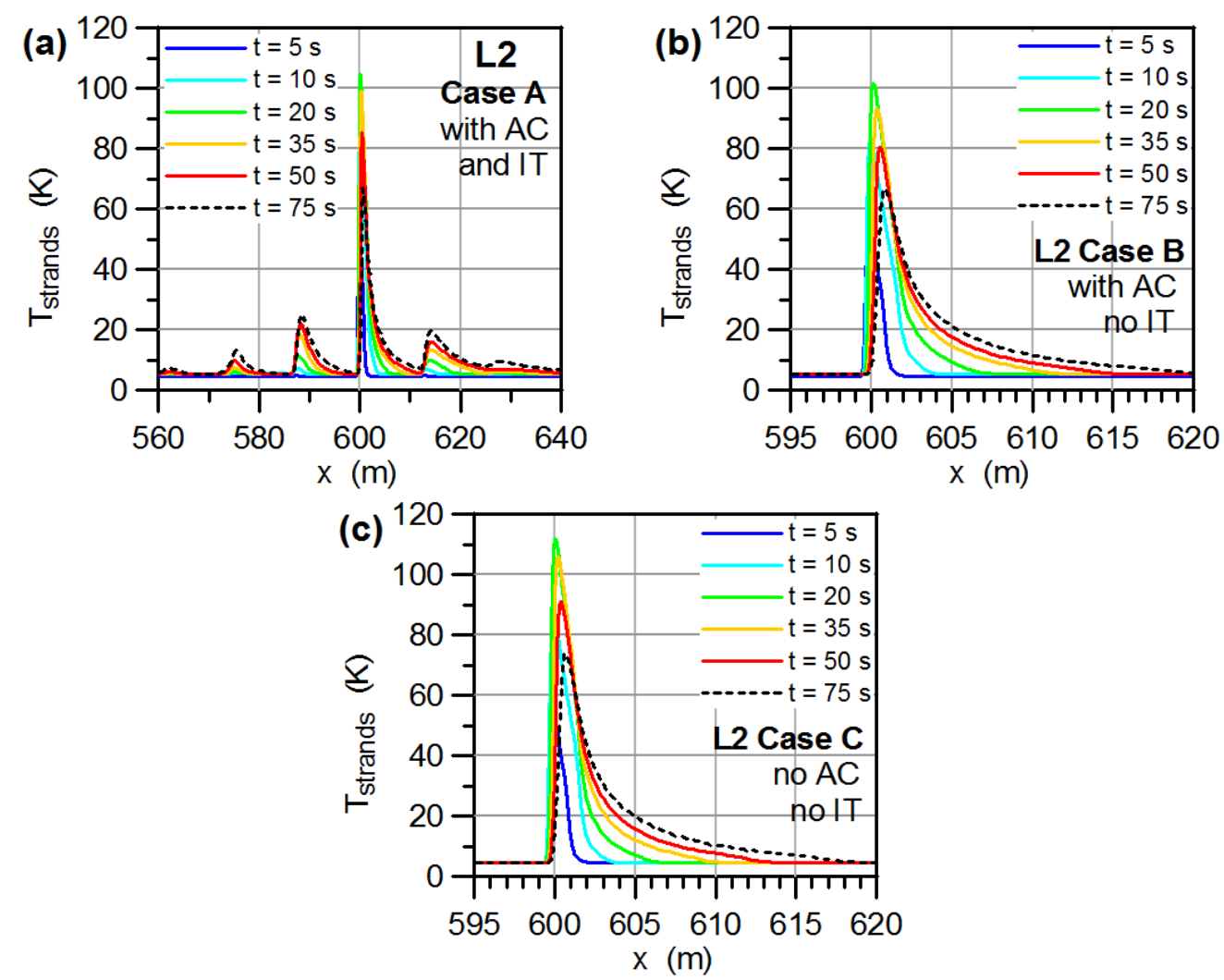

Figure 11. Time evolution of the strands temperature profile during quench in the layer L2.
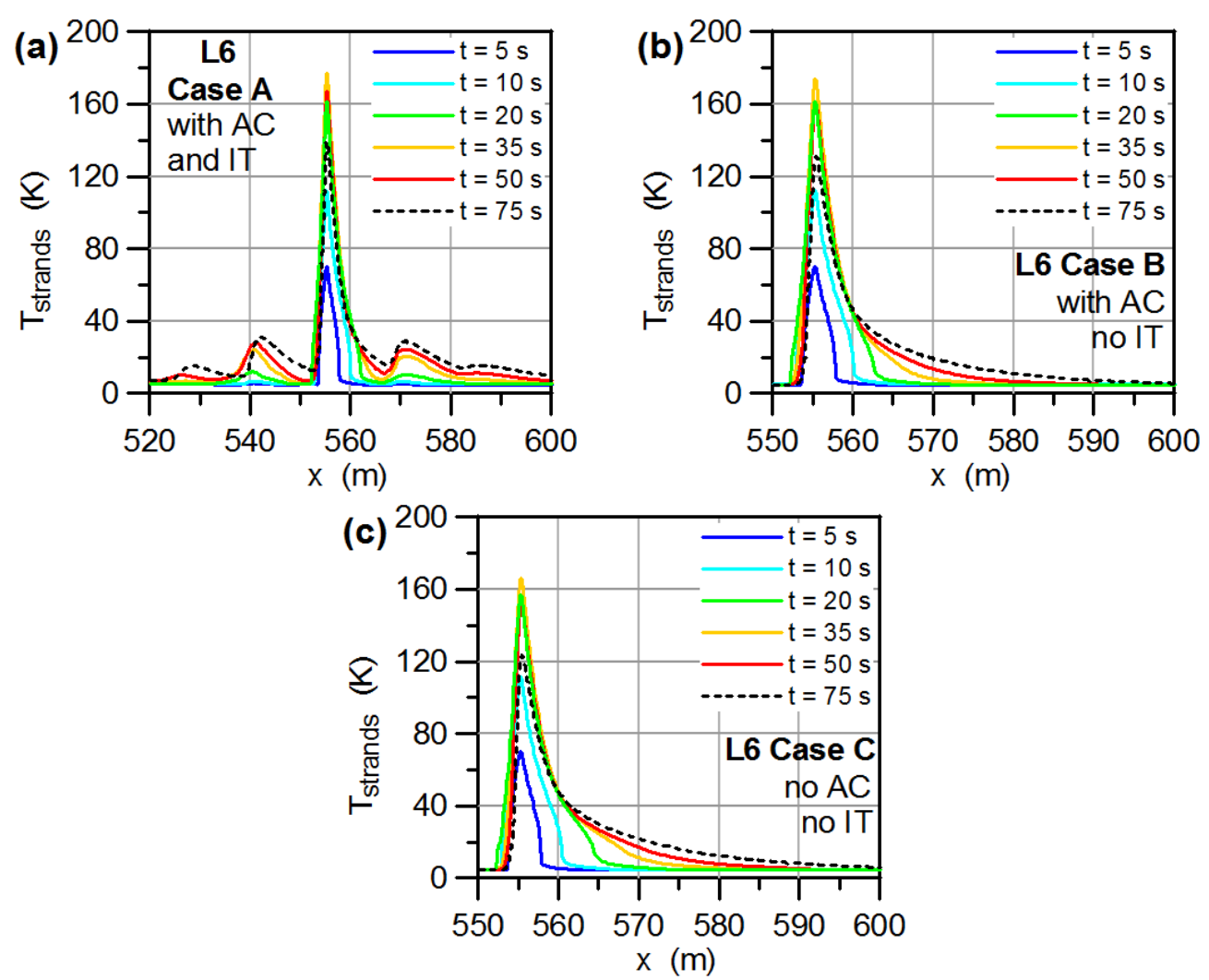

Figure 12. Time evolution of the strands temperature profile during quench in the layer L6. 

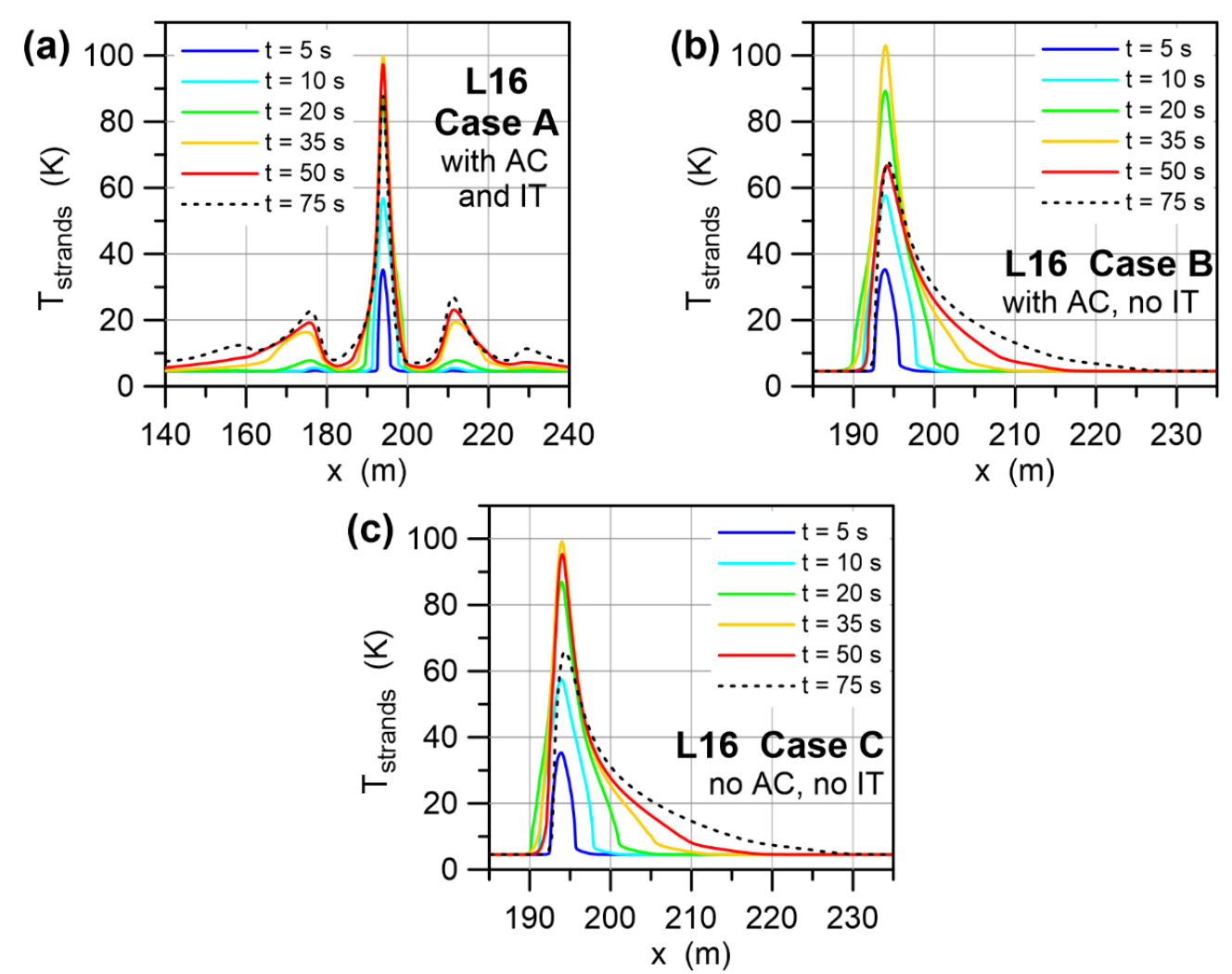

Figure 13. Time evolution of the strands temperature profile during quench in the layer L16.
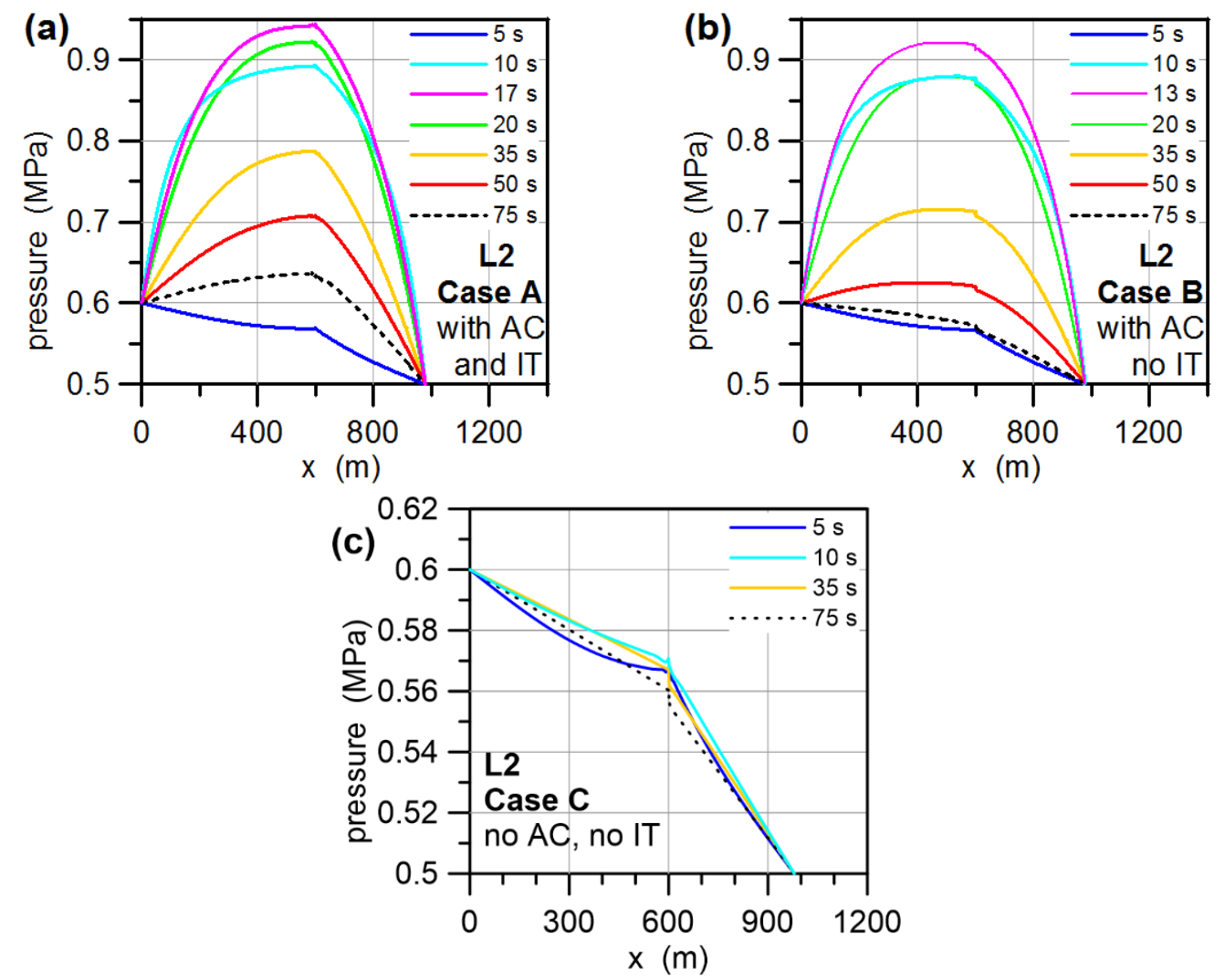

Figure 14. Time evolution of the pressure profile during quench in the layer L2. 

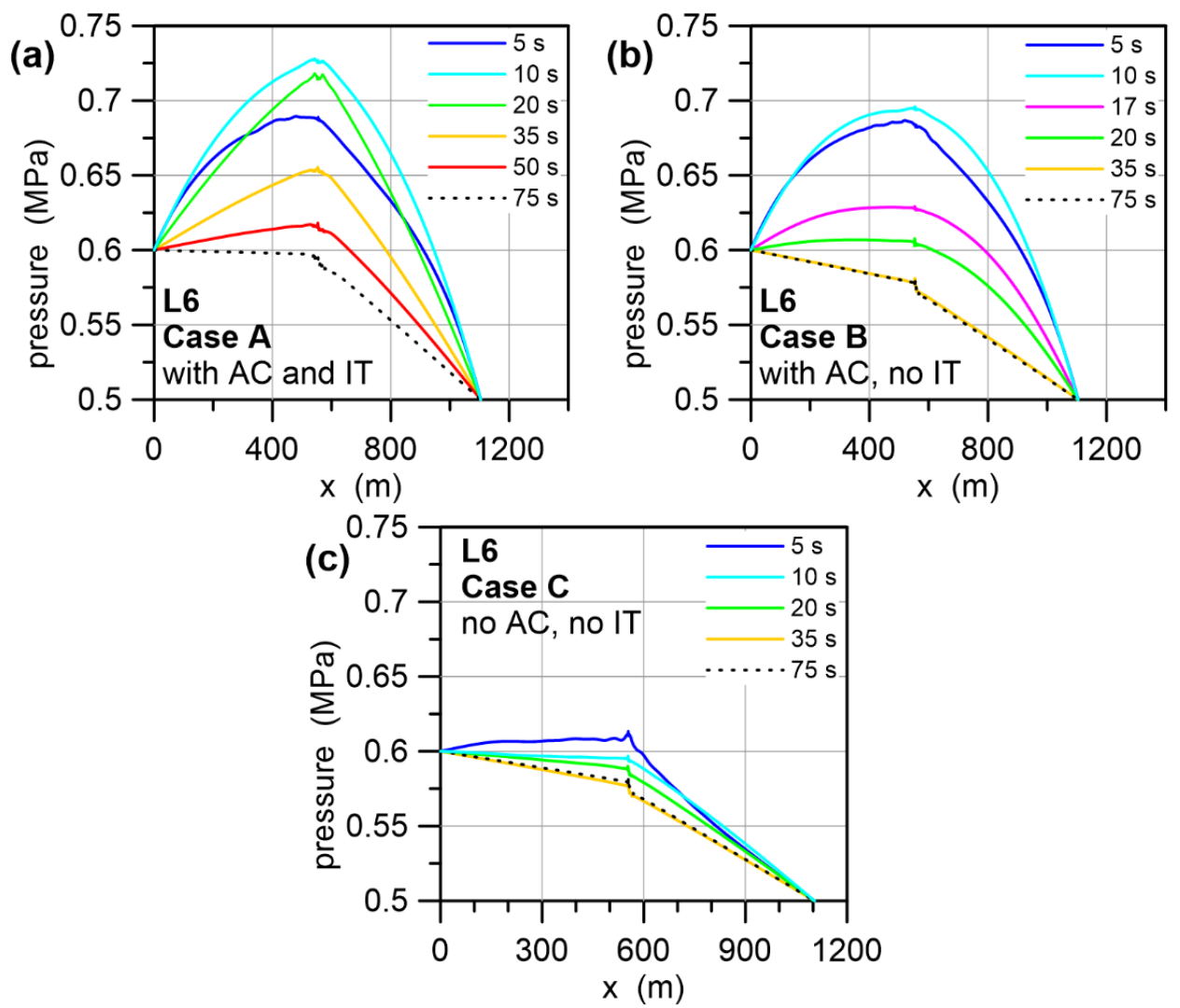

Figure 15. Time evolution of the pressure profile during quench in the layer L6.
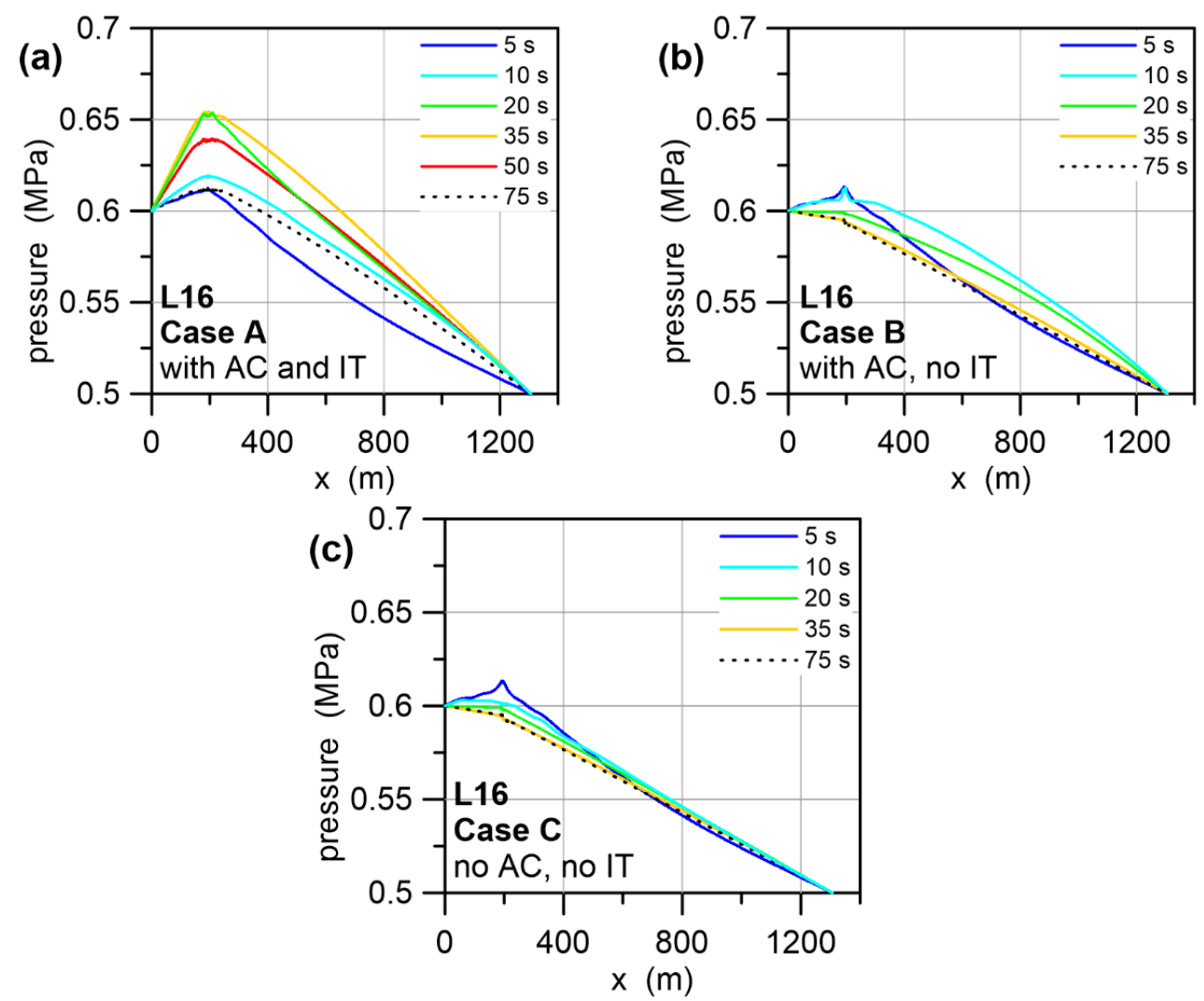

Figure 16. Time evolution of the pressure profile during quench in the layer L16. 

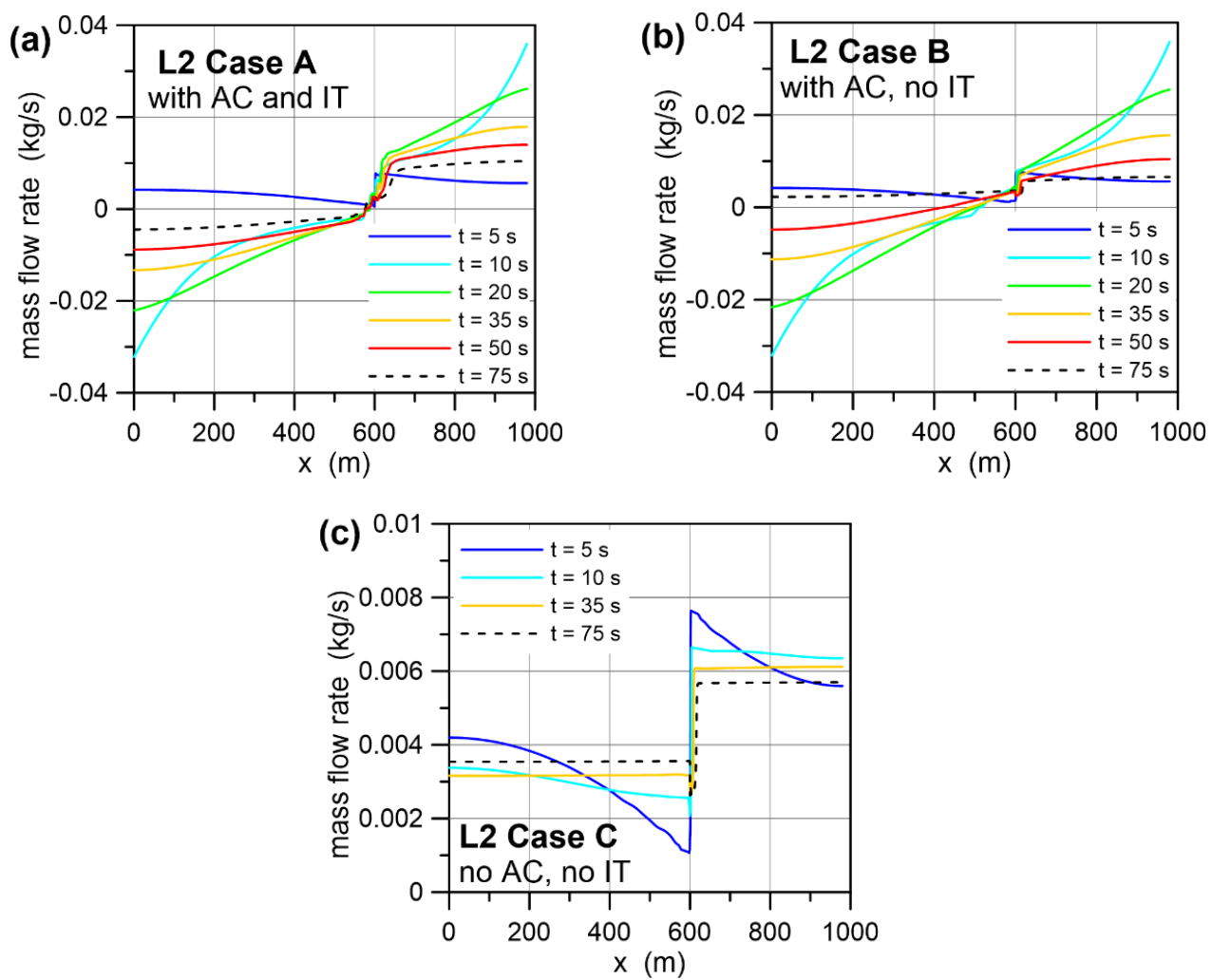

Figure 17. Time evolution of the mass flow rate profile during quench in the layer L2.
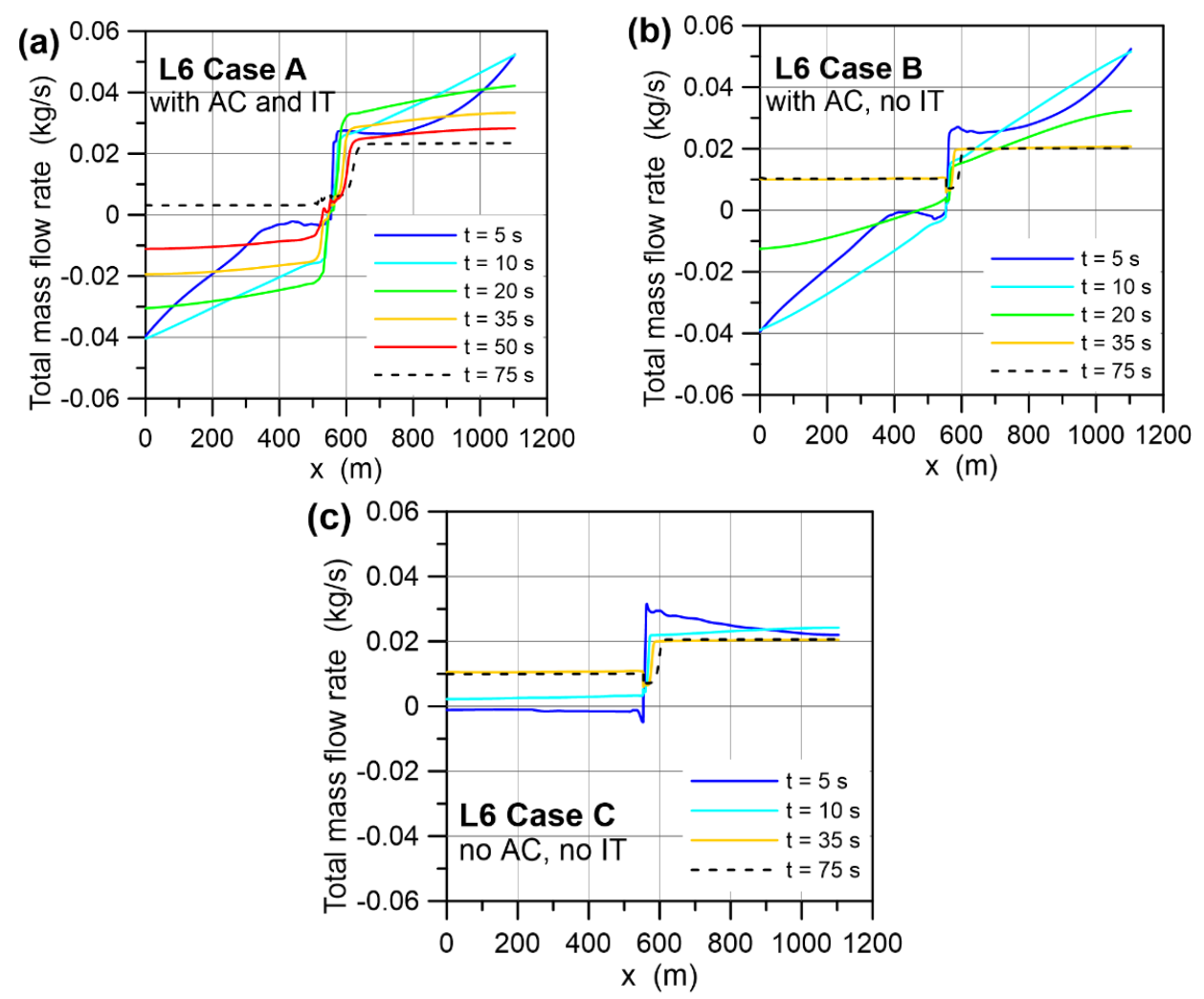

Figure 18. Time evolution of the total mass flow rate (sum of mass flow rates in the bundle region and in all cooling channels) profile during quench in the layer L6. 

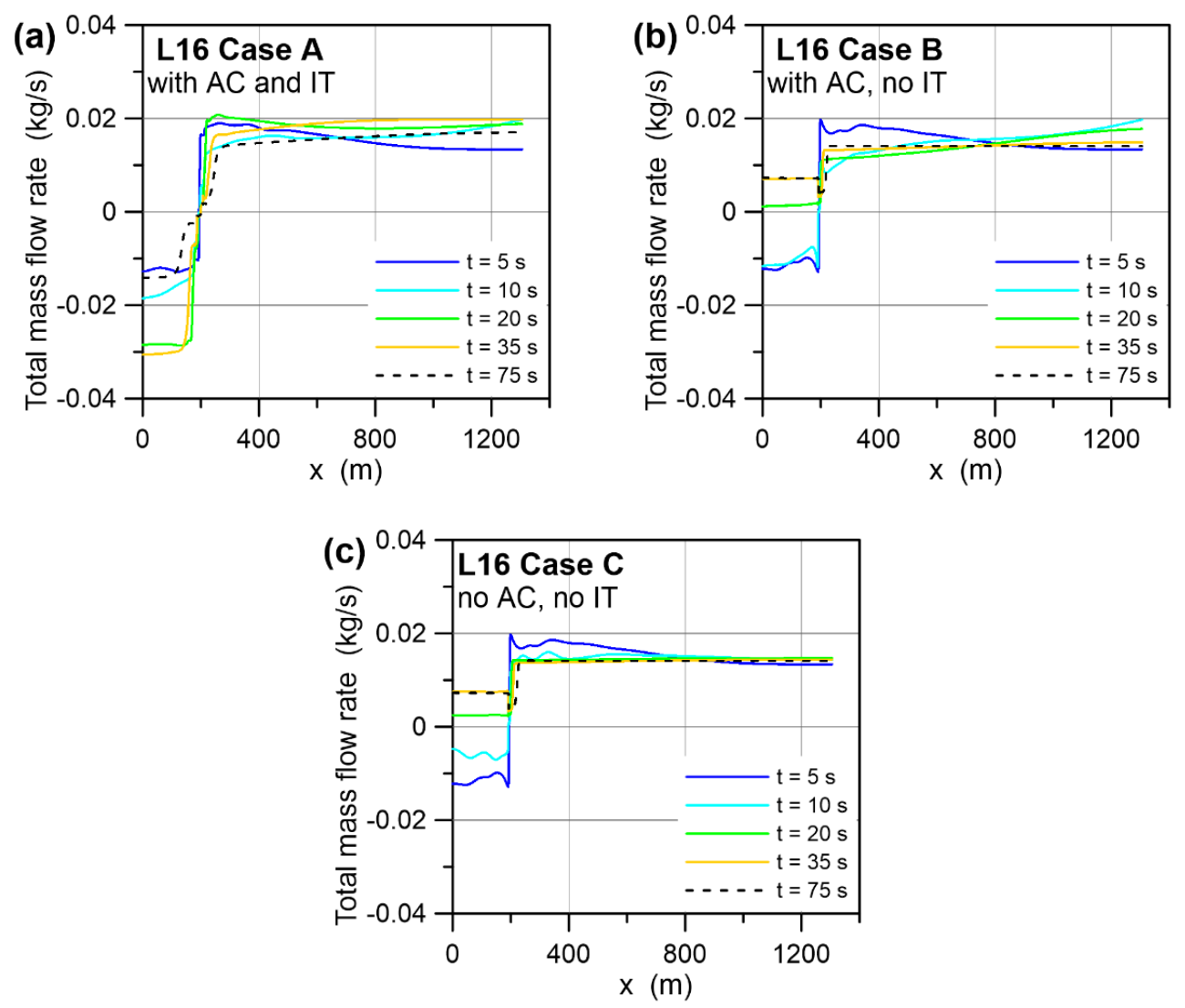

Figure 19. Time evolution of the total mass flow rate (sum of mass flow rates in the bundle region and in all cooling channels) profile during quench in the layer L16.

It is seen than the width of the normal zone in Figs. 12b-c and 13b-c is much larger than in Fig. 11bc, which means that quench propagation in the considered LTS conductors (L6 and L16) is larger than in the L2 HTS conductor. This effect may result mainly from much larger temperature margin in the L2 HTS conductor than in L6 and L16 LTS conductors.

Taking into account IT heat transfer results in a small reduction of $\Delta T_{\text {marg }}$ in the turns adjacent to the location of heat disturbance, which should increase speed of quench propagation, and thus shorten the quench detection time and reduce the hot-spot temperature. However, in the considered layers this effect is not significant (see the $t_{\text {dump }}$ values in Table 1), particularly for L6 in which quench detection is very fast.

IT heat transfer also allows transverse heat removal from the disturbance region to adjacent turns, which is particularly effective for the jacket and explains the fact that the maximum jacket temperature in Case A is always smaller than in Case B (see Figs. 6, 9 and 10). This effect, however, is not very significant. This is because, the thermal conductivity of the conductor insulation material (in Eq. (2)) is relatively small, particularly at the beginning of quench evolution, when the jacket temperature is low ( $\lambda$ varies monotonically in the range $0.06-0.36 \mathrm{~W} /(\mathrm{m} \cdot \mathrm{K}$ ) with the temperature change from 5 to $80 \mathrm{~K}$ ). On the other hand, IT heat transfer results in formation of side maxima of temperature in the turns adjacent to the location of heat disturbance (see Fig. 11a, 12a and 13a) and in pressure rise in these turns, which may lead to formation of a few local pressure maxima in the region of the central hot spot observed e.g. in L6 (see curves for $\mathrm{t}=10 \mathrm{~s}$ and $20 \mathrm{~s}$ in Fig. 15a), which may disturb the mass flow rate in the centre of a normal zone, and as a result may slow down expulsion of heated helium and impede 
longitudinal heat removal from the disturbance region. This effect might explain the observation that the maximum strands temperature in L6 in Case A is higher than in Case B for $t>20 \mathrm{~s}$ (see Figs. 7b and $8 b)$.

Taking into account heat generation due to AC losses during the current dump leads to small temperature rise along the whole conductor. In a normal zone temperature rise due to AC losses may also slightly increase copper resistivity and thus enhance Joule heat generation. So, it could be expected that taking into account AC losses (Cases A and B) should lead to increase of the maximum hot-spot temperature with respect to Case $\mathrm{C}$ (with no AC losses). Such an effect is observed in layers L6 and L16. However, in L2 with the largest AC losses, the observed trend is just the opposite - the maximum temperature of strands and of the jacket is the highest in Case C. This apparently surprising effect may be attributed to significant pressure rise in conductor with the maximum in the central hot-spot region (see Fig.14a-b) which accelerates outflow of heated helium from the hot-spot region in both directions (see Fig. 17a-b), and thus strongly enhances heat removal. Similar significant pressure rise (Fig. 15a-b) and acceleration of helium outflow (Fig. 18a-b) is also observed in L6 conductor. However, in the L6 conductor strands are in direct thermal contact only with helium flowing in the bundle region (H1 in the THEA model, see Fig. 2), where the mass flow rate is very small as compared to the side and rectangular cooling channels ( $\mathrm{H} 2$ and $\mathrm{H} 3$, respectively in the THEA model) due to the very hydraulic impedance in $\mathrm{H} 1$ (the ratio of mass flow rates $\dot{m}_{\mathrm{H} 1} /\left(\dot{m}_{\mathrm{H} 2}+\dot{m}_{\mathrm{H} 3}\right)$ in L6 and L16 is typically in the range $\left.0.01-0.04\right)$. This could be a reason why flow acceleration has smaller cooling effect on strands in L6 than in L2.

\section{Summary conclusion and perspectives}

The performed quench analysis of the DEMO CS1 coil designed by EPFL-SPC shows that in all layers the hot-spot temperatures of the conduit are safely below the acceptable limit of $150 \mathrm{~K}$ [26], however the maximum strands temperature in $\mathrm{Nb}_{3} \mathrm{Sn}$ conductors (L6 - L14) slightly exceeds $150 \mathrm{~K}$, which calls for attention of the coil designers. Taking into account IT heat transfer and heat generation due to AC losses during the fast discharge for the assumed trial value $n \tau=75 \mathrm{~ms}$ has a limited influence on the maximum hot-spot temperature, however it was observed that each of these effects may lead either to lowering or to increasing the maximum hot-spot temperature in different conductors. Thus, it is impossible to decide a priori which of the considered models (Case A, B or C) is the most conservative. Different thermal-hydraulic phenomena related to IT heat transfer and AC losses which may affect the hot-spot temperature have been discussed.

Recently it was suggested in $[28,29]$ that the model of a HTS conductor in which all macro-strands are merged into a single thermal component may not be fully appropriate for quench simulations. On the other hand, modelling of a full size conductor with each HTS macro-strand treated as a separate thermal component might be challenging, taking into account computational capabilities of the THEA code. This issues should be studied in the future.

\section{Acknowledgements}

We would like to thank the anonymous Reviewers for a careful reading of the manuscript, providing many useful suggestions which helped to improve the quality of the paper.

This work has been carried out within the framework of the EUROfusion Consortium and has received funding from the Euratom research and training programme 2014-2018 and 2019-2020 under grant agreement No 633053. The views and opinions expressed herein do not necessarily reflect those of the European Commission. This scientific work was partly supported by Polish Ministry of Science and Higher Education within the framework of the scientific financial resources in the year 2019 allocated 
for the realization of the international co-financed project (contract No. 5011/H2020EURATOM/2019/2 of 20 December 2019).

\section{References}

[1] Donné T, et al. European Research Roadmap to the Realisation of Fusion Energy (long version) http://oldweb.reak.bme.hu/fileadmin/user_upload/felhasznalok/pokol/Oktatas_2018_2019_1/20 18 Research roadmap long_version.pdf, 2018.

[2] Federici G, et al. Overview of the design approach and prioritization of R\&D activities towards an EU DEMO. Fus Eng Des 2016; 109-111, Part B: 1464-74.

[3] Federici G, et al. DEMO design activity in Europe: Progress and updates. Fus Eng Des 2018; 136: 729-41.

[4] Zani L, et al. Overview of Progress on the EU DEMO Reactor Magnet System Design. IEEE Trans Appl Supercond 2016; 26: Art. no. 4204505.

[5] Corato V, et al. Progress in the design of the superconducting magnets for the EU DEMO. Fusion Eng Des 2018; 136: 1597-1604.

[6] Sedlak K, et al. Advance in the conceptual design of the European DEMO magnet system, Supercond Sci Technol 2020; 33: Art. No. 044013.

[7] Vallcorba R., et al. Thermohydraulic Analyses on CEA Concept of TF and CS Coils for EUDEMO. IEEE Trans Appl Supercond 2018; 28: Art. no. 4202605.

[8] Torre A, Ciazynski D, Zani L. EU-DEMO TF and CS Magnet Systems Design and Analyses Performed at CEA. IEEE Trans Appl Supercond 2017; 27: Art no. 4900705.

[9] Nunio F, Torre A, Zani L. Mechanical analysis of the European DEMO central solenoid pre-load structure and coils. Fus Eng Des 2019; 146 Part A: Pages 168-72.

[10] Zani L, et al. Status of CEA Magnet Design Tools and Applications to EU DEMO PF and CS Magnets. IEEE Trans Appl Supercond 2018; 28: Art no. 4902205.

[11] Wesche R, Bykovsky N, Sarasola X, Sedlak K, Stepanov B, Uglietti D, Bruzzone P. Central solenoid winding pack design for DEMO. Fus Eng Des 2017; 124: 82-85.

[12] Wesche R, Sarasola X, Sedlak K, Bykovsky N, Stepanov B, Uglietti D, Bruzzone P. DEMO central solenoid design based on the use of HTS sections at highest magnetic field. IEEE Trans Appl Supercond 2018; 28: Art. no. 4203605.

[13] Dembkowska A, Lewandowska M, Sarasola X. Thermal-Hydraulic Analysis of the DEMO CS Coil. IEEE Trans Appl Supercond 2018; 28: Art. no. 4205605

[14] Dembkowska A, Lewandowska M, Sarasola X. Helium flow and temperature distribution in the CS1 module of the DEMO CS coil. Proc Int Conf on Electromagnetic Devices and Processes in Environment Protection joint with Seminar Applications of Superconductors, Nałęczów, Poland, 2017, doi: 10.1109/ELMECO.2017.8267759

[15] Savoldi L, Bonifetto R, Zanino R. Thermal-hydraulic analysis of the 2016 design of a DEMO TF magnet, CS WP and PF WP. Final report on MAG-2.2-T011, https://idm.eurofusion.org/?uid=2NBFY9

[16] Dembkowska A, Lewandowska M, and Sarasola X. Thermal-hydraulic analysis of the upgraded EU-DEMO CS1 coil. Proc. SPIE 11054, Superconductivity and Particle Accelerators, Krakow, Poland, 2018: Art. no. 1105409, doi: 10.1117/12.2525336. 
[17] THEA-Thermal, Hydraulic and Electric Analysis of Superconducting Cables. User's Guide Version 2.3, CryoSoft, 2016, https://supermagnet.sourceforge.io/manuals/Thea_2.3.pdf

[18] Corato V, et al. Common operating values for DEMO magnets design for 2016. http://www.eurofusionscipub.org/archives/eurofusion/common-operating-values-for-demo-magnets-design-for2016-2.

[19] Savoldi L and Zanino R. Common approach for thermal-hydraulic calculations, Memo for WPMAG-2.1-D01, 2016. https://idm.euro-fusion.org/?uid=2LMECE .

[20] Shah RK, Sekulić DP. Fundamentals of Heat Exchanger Design, Wiley, New Jersey, 2003 (pp 476-83).

[21] Takahata K, Tamura H, Mito T. Thermal contact conductance between the bundle and the conduit in cable-in-conduit conductors. IEEE Trans Appl Supercond 2004; 14: 1477-80.

[22] Bagnasco M, Bottura L, Lewandowska M. Friction factor correlation for CICC's based on a porous media analogy. Cryogenics 2010; 50: 711-9.

[23] Heller R, Gade PV, Fietz WH, Vogel T, Weiss K-P. Conceptual design improvement of a Toroidal Field coil for EU DEMO using High-Temperature Superconductors. IEEE Trans Appl Supercond 2016; 26: Art. No. 4201105.

[24] ANSYS Academic Research Mechanical, Release 17.0, Available: http://www.ansys.com/products/

[25] Savoldi L, et al. Quench Propagation in a TF Coil of the EU DEMO. Fus Sci Technol 2017; 72: $439-48$.

[26] ITER Design Description Document. Magnets. Section 7: Conductors 2009 ITER_D_2NBKXY v1.2

[27] Lewandowska M, Dembkowska A, Heller R, Wolf M. Thermal-hydraulic analysis of an HTS DEMO TF coil. Cryogenics 2018; 96:125-32.

[28] Zappatore A, Fietz WH, Heller R, Savoldi L, Wolf MJ, Zanino R. A critical assessment of thermal-hydraulic modeling of HTS twisted-stacked-tape cable conductors for fusion. Supercond. Sci. Technol. 2019; 32: Art. No. 084004.

[29] Wolf M, Heller R, Fietz WH, Weiss K-P. Design and analysis of HTS subsize-conductors for quench investigations towards future HTS fusion magnets. Cryogenics 2019; 104: Art. No. 102980 